\title{
Klassische Implantate, die man kennen sollte: der Plattenspanner
}

Nadine Diwersi, Frank J. P. Beeres, Reto Babst, Björn-Chritian Link

\begin{abstract}
Ziel der operativen Frakturversorgung ist die Wiederherstellung der Funktionalität durch möglichst genaue Rekonstruktion der Anatomie und Ermöglichung einer frühfunktionalen Mobilität. Um dies zu bewirken, ist die Kenntnis der Grundprinzipien der Frakturversorgung unabdingbar. Ist das gewünschte Resultat nicht mit den üblichen Hilfsmitteln erreichbar, kann das Wissen um alternativ einzusetzende Instrumente und Implantate von Vorteil sein. Ein solches Instrument ist der Plattenspanner, ein zwar in die Jahre gekommenes, jedoch hilfreiches Instrument für die Applikation von kontrollierter Kompression und Distraktion in der Frakturversorgung oder bei Osteotomien. Auf ihn soll im Folgenden näher eingegangen werden.
\end{abstract}

\section{Implantate und Instrumente der Osteosynthese: ein geschichtlicher Auszug}

In den Anfängen der Frakturversorgung kamen vor allem externe Vorrichtungen und Extensionsapparate zum Einsatz. So verwendeten die Ägypter vor 5000 Jahren Tuchbandagen und Palmenrinden, andere hingegen Kalk mit Eiweiß vermischt zur externen Schienung. Noch heute finden Bambusplatten in einigen Ländern Verwendung bei der Frakturruhigstellung und zeigen dabei gute Ergebnisse [2].

Erste Platten- und Schraubenkonstruktionen verwendete Carl Hansmann, ein deutscher Chirurg, der als einer der Pioniere auf dem Gebiet der Osteosynthese gilt, im Jahre 1886 [1]. Einer der größten Vorreiter moderner innerer und äußerer Knochenschienung ist der belgische Chirurg Albin Lambotte, Chirurg wie Mechaniker, der fast all seine Instrumente und Implantate in der hauseigenen Werkstatt selbst herstellte und unter anderem den Fixateur externe entwickelte [4]. Robert Danis, ebenfalls ein belgischer Arzt, entwickelte 1938 den „Coapteur“, eine Platte, die eine axiale Kompression ermöglichte und damit die Voraussetzung für die interfragmentäre Kompression [3] schuf.

Mit der Gründung der Arbeitsgemeinschaft für Osteosynthesefragen im Jahr 1958 und der hiermit initiierten Entwicklung von geeigneten Implantaten und Instrumenten für eine sichere und reproduzierbare operative Knochenbruchbehandlung wurde auch der sogenannte Plattenspanner, eine Weiterentwicklung des Coapteurs von
Robert Danis, zur Erreichung einer interfragmentären Kompression im Frakturspalt entwickelt ( $\mathbf{A} \mathbf{A b} \mathbf{b}$. 1). Die absolute Stabilität war in der Pionierphase der operativen Frakturbehandlung ein wichtiges Axiom zur Ermöglichung einer frühfunktionellen Behandlung. Interfragmentäre Kompression wurde primär durch die Verwendung von Zugschrauben erreicht. Die erzielte Kompression wurde dann durch eine Neutralisationsplatte, die den Frakturspalt vor Torsions-, Biege- und Scherkräften schützte, gehalten. Bei Querfrakturen oder kurzen Schrägfrakturen bzw. Osteotomien im Schaftbereich konnte aber eine interfragmentäre Kompression nur durch einen Plattenspanner appliziert werden. Mit der Weiterentwicklung von Platten, die eine exzentrische Bohrung zuließen (dynamische Kompressionsplatten [DCP]) war die absolute Notwendigkeit zur Verwendung des Plattenspanners nicht mehr gegeben. Trotzdem gibt es vor allem in der Chirurgie von Pseudarthrosen und im Rahmen von Osteotomien immer wieder Situationen, in denen kontrollierte interfragmentäre Kompression angewandt werden muss. Dafür eignet sich der Plattenspan-

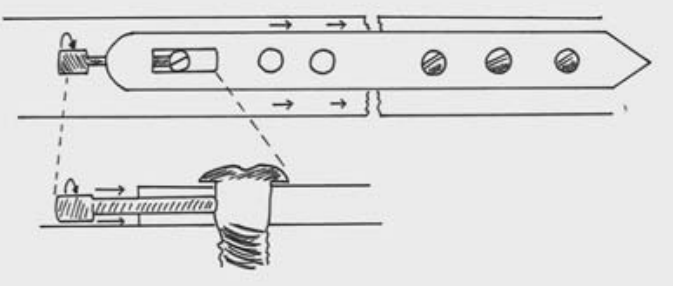

- Abb. 1 Der Coapteur von Robert Danis. 


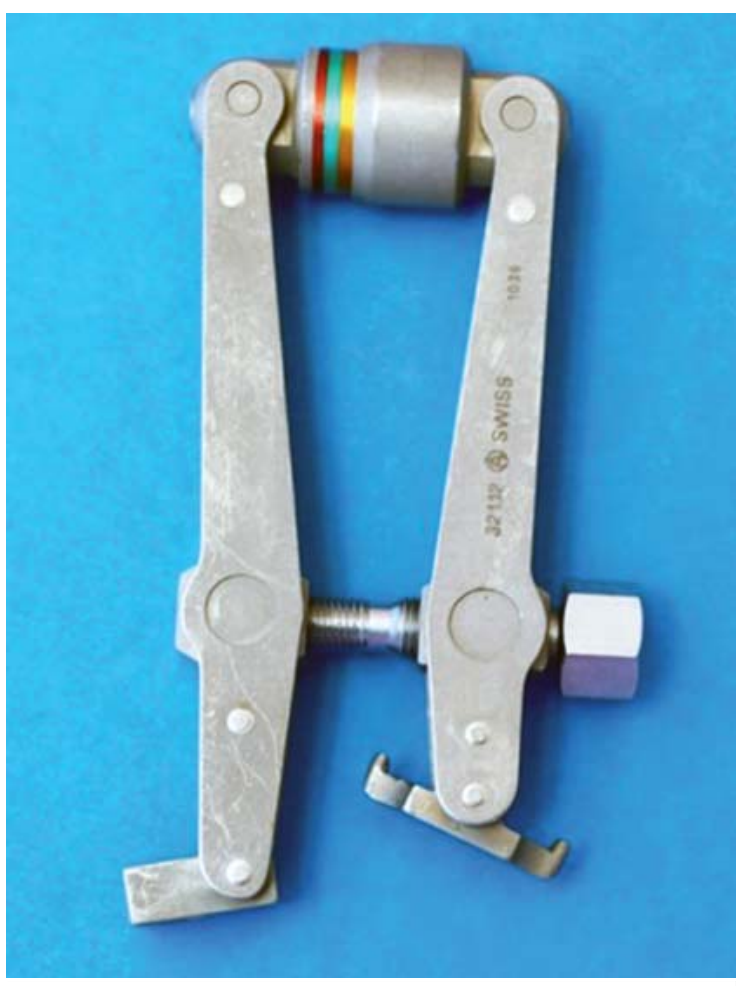

- Abb. 2 Beispiel eines Plattenspanners. Der gelbe Ring im Querarm steht für eine Kompressionskraft von 050 kp, der grüne Ring für 50-100 kp, der rote für > $100 \mathrm{kp}$.

ner der AO (Arbeitsgemeinschaft für Osteosynthesefragen).

\section{Einsatzmöglichkeiten des Plattenspanners}

Wie der Coapteur zielt auch der Plattenspanner auf interfragmentäre Kompression ab, kann aber auch zur Distraktion und somit als Repositionshilfe eingesetzt werden. Der Plattenspanner kann hilfreich sein, wenn größere Frakturlücken geschlossen werden müssen. Bei Verwendung von DCP-Platten oder rohrförmigen Platten (1/4-, 1/3oder $1 / 2$-Rohr-Platte) empfiehlt sich die Verwendung des Plattenspanners nur, wenn Kompressionskräfte größer als $100 \mathrm{kp}$ erzeugt werden müssen oder der Spannweg über $2 \mathrm{~cm}$ beträgt [6].

Der Plattenspanner sollte insbesondere dann in Betracht gezogen werden, wenn die bei quer verlaufenden oder kurzen Schrägfrakturen verwendeten Platten durch exzentrische Bohrung im DCP-Plattenloch nicht genügend Kompression erzeugen, um den Frakturspalt zu schließen. Die meisten AO-Platten sind mit dem Plattenspanner kompatibel. Teilweise haben die Platten im Endloch eine kleine Anfräsung für den Haken des Plattenspanners. Der Haken kann jedoch auch im Endloch selbst eingehakt werden.

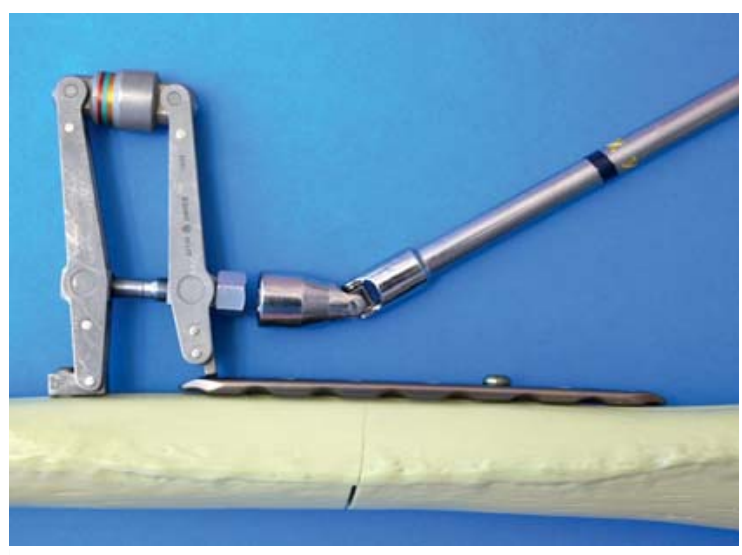

- Abb. 3 Der Plattenspanner im Einsatz am Beispiel eines Kunstknochens.

Ist das Ziel die Kompression, öffnet man den Plattenspanner vollständig und platziert den Haken im letzten Plattenloch. Über eine kurze monokortikale Schraube wird anschließend die Fußplatte des Plattenspanners am Knochen befestigt. Mittels Ringschlüssel kann dann die Spannschraube am Plattenspanner angezogen werden, worauf der Plattenspanner die Platte zu sich zieht und hierdurch Kompression auf die Fraktur- oder Osteotomiezone kommt. Farbige Ringe im Querarm des Plattenspanners zeigen dabei die Kompressionskraft an, welche auf die Frakturzone ausgeübt wird. Der gelbe Ring steht dabei für eine Kompressionskraft von 0-50 kp, der grüne Ring für 50-100 kp, der rote für > 100 kp ( Abb. 2).

Ist Distraktion das Ziel, muss der Plattenspanner geschlossen werden. Der umgedrehte Haken wird in eine kleine, endständige Kerbe in der dynamischen Kompressionsplatte eingehakt und die Fußplatte des Spanners wiederum mittels monokortikaler Schraube ossär befestigt. Öffnet man nun die Spannschraube am Plattenspanner, wird die Fraktur- oder Osteotomiezone distrahiert ( Abb. 3).

Stark osteoporotische Frakturen, wie sie bei älteren Patienten im Rahmen proximaler Femurfrakturen zu finden sind, können bei der Frakturversorgung von der Verwendung eines Plattenspanners profitieren, und so kann über die Reposition, Kompression und Plattenspannung eine lastteilende Versorgung hergestellt werden [5].

Bei reinen Querfrakturen gilt es zu beachten, dass bei Spannung einer geraden Platte nur die plattennahe Kortikalis unter Druck kommt und die Platte auf Biegung beansprucht wird. Biegt man die Platte in der Mitte vor und setzt nach Einbringen einer ersten Schraube den Plattenspanner an, so kommt es zunächst zu einem Auseinanderklaffen der plattennahen Kortikalis und zum Kontakt der plattenfernen Kortikalis. Durch das Anziehen 


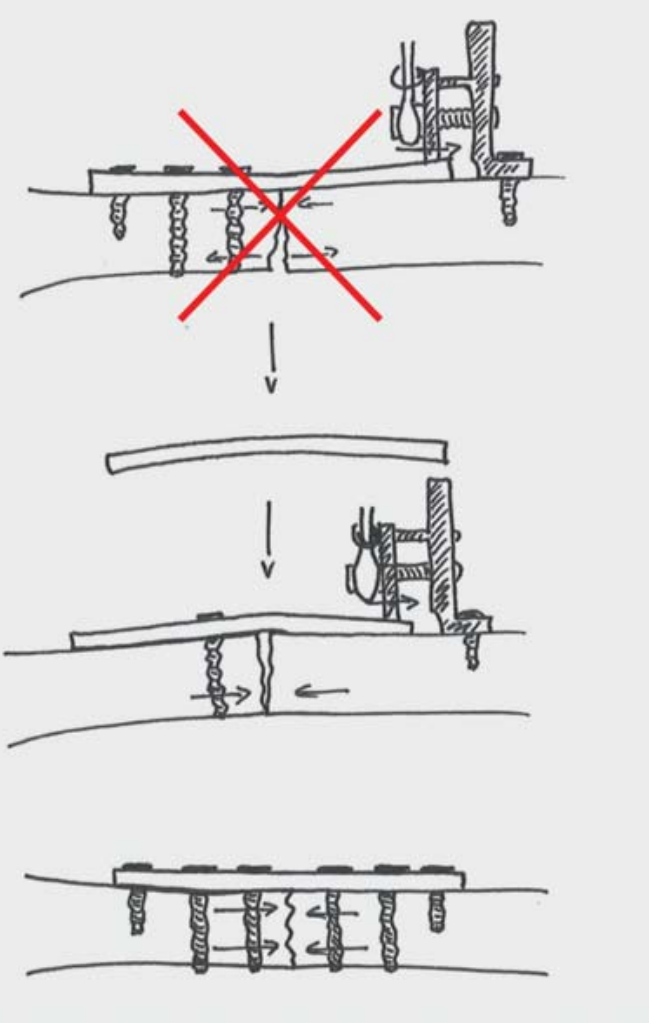

- Abb. 4 Prinzip der vorgebogenen Platte bei Querfrakturen unter Verwendung des Plattenspanners.

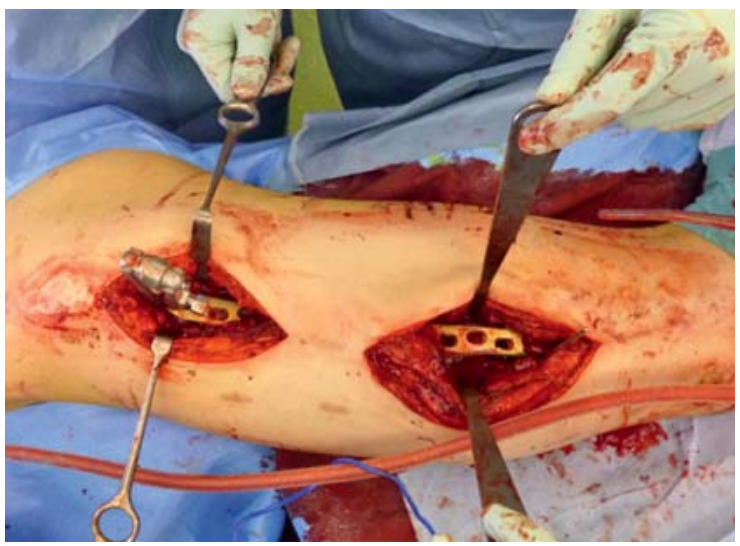

- Abb. 6 Der Plattenspanner im Einsatz bei einer hypertrophen Pseudarthrose mit Notwendigkeit der Schließung des Pseudarthrosespalts von $>1 \mathrm{~cm}$. Initial war aufgrund des engen Femurmarkkanals eine Plattenosteosynthese in minimalinvasiver Technik durchgeführt worden. Bei Pseudarthrose erfolgte eine Revision mit Wellenplatte und Spongiosaplastik bei dem Patienten, der nebenbefundlich mit Drogen- und Nikotinabusus auffiel. Abschließend dann die oben genannte Operation mit Verwendung des Plattenspanners. Vgl. Rx. Juli 2016 • Abb. 7.

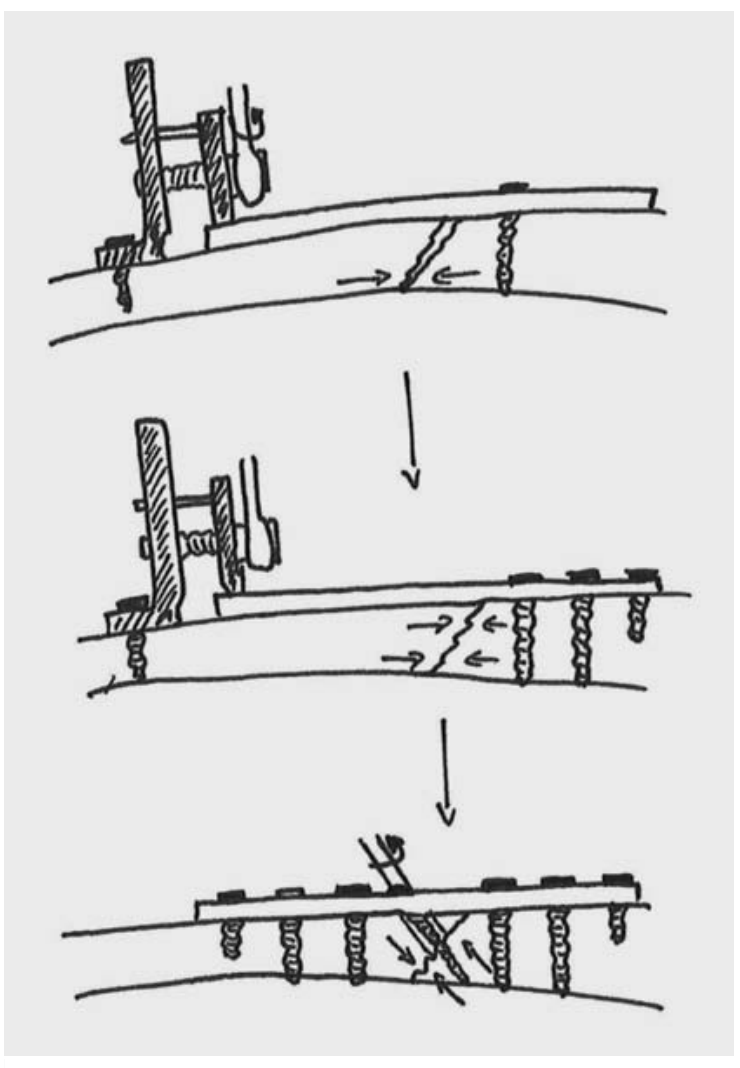

- Abb. 5 Verwendung des Plattenspanners bei Radiusquerfrakturen.

der Spannschraube am Plattenspanner wird entlang der gesamten Frakturzone Druck erzeugt und der Knochen begradigt sich ( $\boldsymbol{A}$ Abb. $\mathbf{4}$ und $\mathbf{5}$ ). Diese Technik wird unter anderem bei der operativen Versorgung von Querfrakturen oder Pseudarthrosen nach konservativem oder nicht erfolgreichem operativem Therapieversuch verwendet [7] ( $\triangleright$ Abb. 6 und 7).

Auch im Rahmen der Versorgung von Radiusschaftschrägfrakturen kann mittels Plattenspanner die Fraktur reponiert und komprimiert werden. Hierzu wird an einer schrägen Fraktur eine leicht überkonturierte Platte zunächst an dem plattenspannerfernen Fragment verschraubt. Nach Anbringen des Plattenspanners nach oben beschriebener Technik wird dieser angespannt und hierüber die Fraktur reponiert. Zusätzlich kann der Frakturspalt bei liegendem und angezogenem Plattenspanner mit einer interfragmentären Zugschraube zusätzlich unter Kompression gebracht werden ( $\mathbf{A b b}$. 5).

Einige Autoren propagieren den Einsatz des Plattenspanners bei der Versorgung von Klavikulapseudarthrosen, während andere aufgrund der geschwungenen Knochenform der Klavikula eher davon abraten, um eine zusätzliche Weichteilexposition zu vermeiden [9]. Zudem könne keine direkte axiale Zugwirkung erreicht werden [8]. 

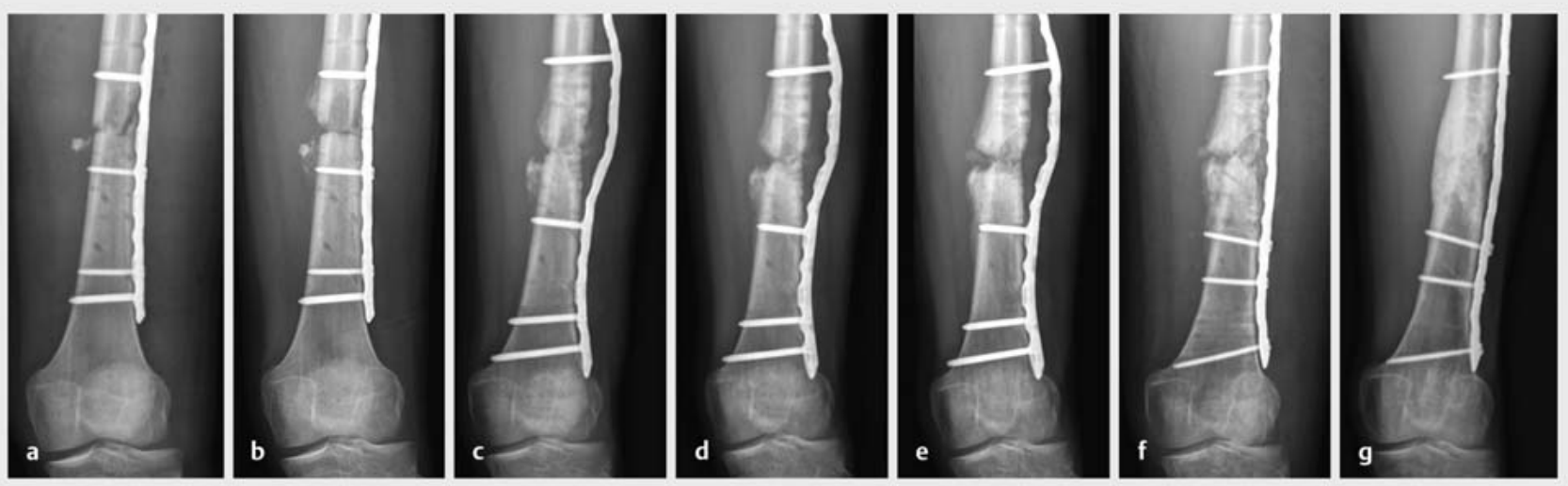

- Abb. 7 Anterior-posteriore Verlaufsröntgenaufnahmen des oben in \ Abb. 6 gezeigten Beispielpatienten von 2015-2018 mit multiplen Revisionseingriffen. Operationsbild Revision Juli 2015.

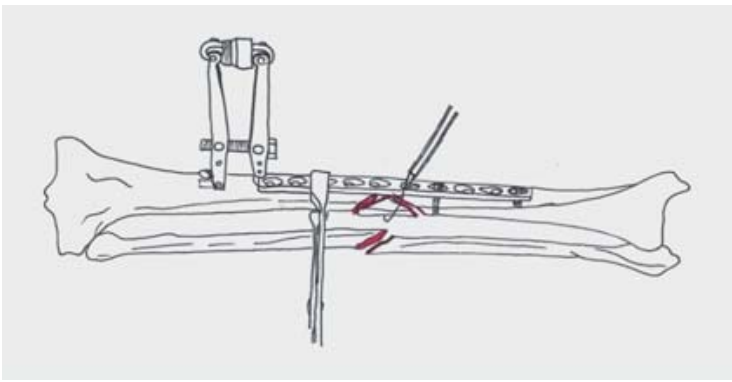

Abb. 8 Verwendung des Plattenspanners zur Distraktion und Säuberung des Frakturspalts.

Zur Distraktion kann der Plattenspanner beispielsweise bei Fibulatrümmerfrakturen verwendet werden, wobei er als Repositionshilfe verwendet wird. Hierzu muss der Spannzahn des Plattenspanners umgekippt werden und die Spannschraube gelockert werden, nachdem diese vor Installation geschlossen wurde [10]. Auch bei schwierig zu säubernden Frakturspalten kann der Plattenspanner durch passageres Aufhalten der interfragmentären Zone verwendet werden ( $\bullet$ Abb. 8).

\section{Alternativen zum Plattenspanner}

Die Hauptaufgabe des Plattenspanners ist wie oben beschrieben die interfragmentäre Kompression. Steht kein Plattenspanner zur Verfügung, so kann die Kompression meist in anderer Technik erzielt werden. Klassischerweise wird hierfür bei entsprechendem Frakturverlauf eine Zugschraube verwendet. Diese verläuft senkrecht zur und durch die Fraktur. Dabei entspricht der Durchmesser des schraubenkopfnahen Gleitlochs dem Durchmesser des Schraubenschafts. Schraubenkopffern wird in einem kleineren Durchmesser gebohrt und die Zugschraube kann greifen, wobei es zum Aufeinanderpressen der Frag-

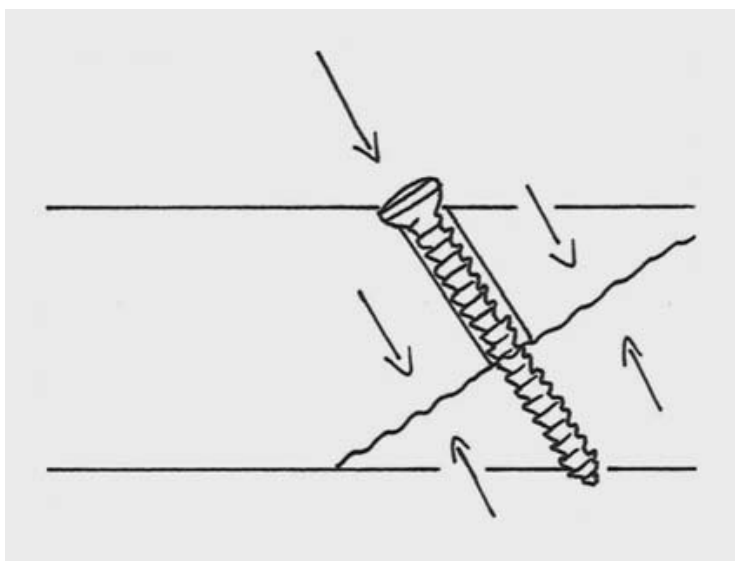

- Abb. 9 Prinzip der Zugschraube.

mentflächen kommt ( $\bullet$ Abb. 9). Bei Querfrakturen ist die Verwendung einer Zugschraube nicht möglich.

Eine weitere Möglichkeit zur Erzeugung interfragmentärer Kompression besteht in der Verwendung von Platten nach dem dynamischen Kompressionsprinzip ( $\bullet$ Abb. 10). Diese Platten verfügen über elliptisch geformte Schraubenlöcher, die symmetrisch - Kompression in beide Richtungen - oder asymmetrisch - Kompression in nur eine Richtung - angeordnet sein können. Setzt man die Schraube bei dieser Art von Platten exzentrisch, so gleitet sie während des Anziehens abwärts und seitlich und führt hierdurch zu einer Kompression des Frakturspalts. Ist keine Kompression gewünscht, kann die Schraube zentral gesetzt werden ( $\bullet$ Abb. 11).

Auch die im eigenen Haus verwendete, leicht aufgebogene Weber-Zange mit Feinsperre bildet eine Alternative zum Plattenspanner. Mit den Branchen der Weber-Zange kann man auf der einen Seite in die Platte, auf der ande- 


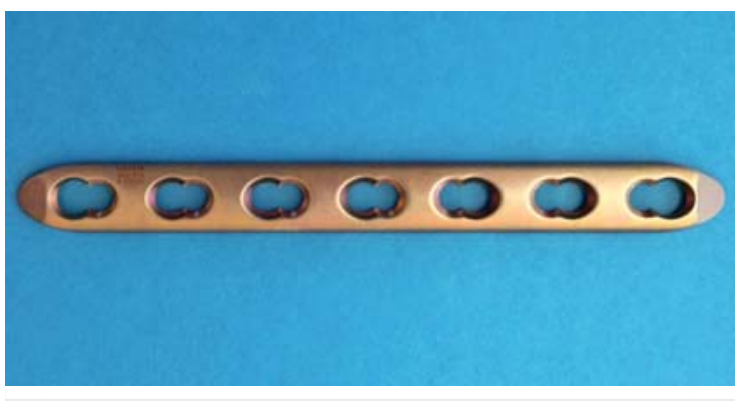

- Abb. 10 Beispiel einer Platte mit dynamischem Kompressionsprinzip (DCP).

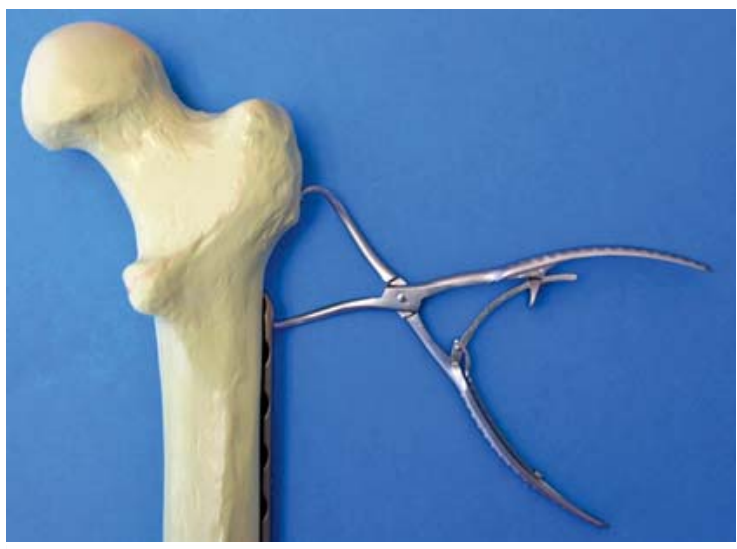

Abb. 12 Die aufgebogene Weber-Zange mit Feinsperre als Alternative zum Plattenspanner.

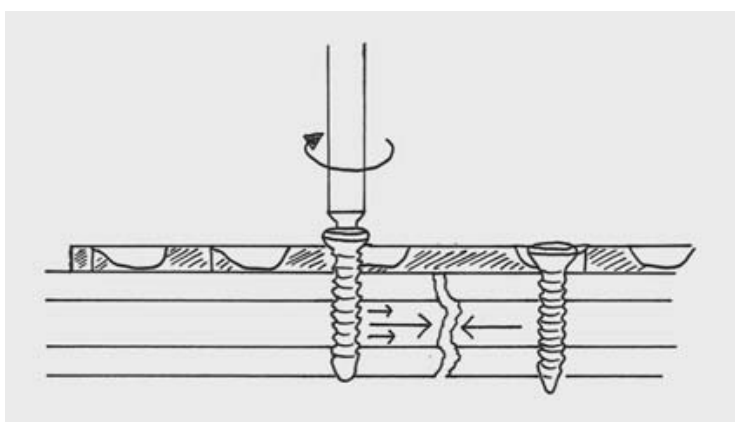

- Abb. 11 Prinzip der dynamischen Kompression über elliptische Schraubenlöcher.

ren Seite in ein 2-mm-Bohrloch greifen und durch Verschließen der Weber-Zange Kompression erzeugen ( $\bullet$ Abb. 12).

Reine Querfrakturen können auch durch die aufgebogene Weber-Zange und ein 1,8-mm-Bohrloch je Frakturseite interfragmentär komprimiert werden ( $\bullet \mathbf{A b b} .13)$.

\section{Zusammenfassung}

Der Plattenspanner wurde in Grundzügen bereits 1938 von Robert Danis in Form des Coapteurs entwickelt und gehört somit zum ursprünglichen Instrumentarium der Osteosynthesetechnik. Er findet heute nur noch wenig Verwendung, da interfragmentäre Kompression häufig bei Querfrakturen oder kurzen Schrägfrakturen durch exzentrisches Bohren bei vorgebogenen Platten mit DCPLöchern appliziert werden kann. Allerdings erlaubt der Plattenspanner insbesondere im Rahmen von Revisions-

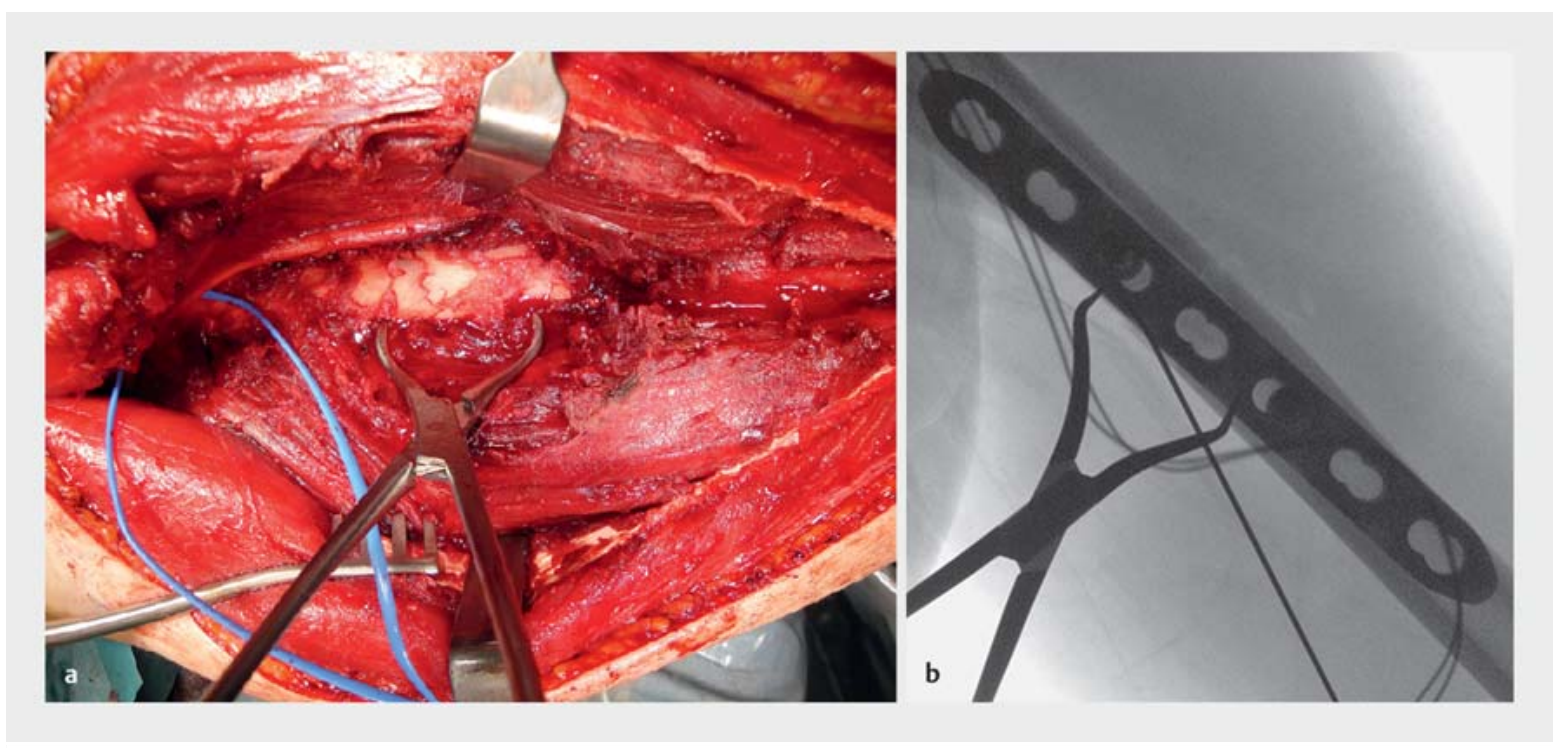

- Abb. 13 Verwendung der aufgebogenen Weber-Zange bei einer reinen Querfraktur. 
eingriffen, bei Korrekturosteotomien oder Pseudarthroseoperationen eine kontrollierte und dosierte interfragmentäre Kompression bei der Notwendigkeit, einen Fraktur-/Pseudarthrose- oder Osteotomiespalt $>1 \mathrm{~cm}$ sicher zu schließen. Daher ist er auch heute noch ein Werkzeug, das im unfallchirurgisch/orthopädischen Repertoire bekannt bleiben sollte.

\section{Interessenkonflikt}

Die Autoren geben an, dass kein Interessenskonflikt besteht.

\section{Autorinnen/Autoren}

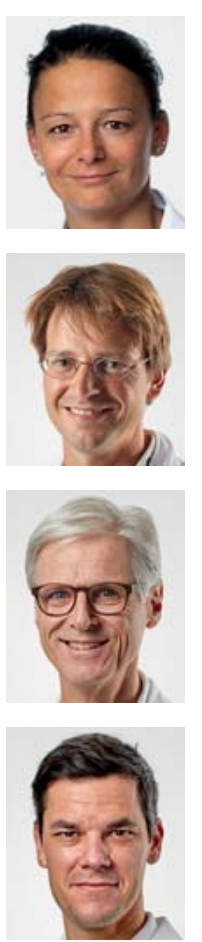

\section{Nadine Diwersi}

Dr. med. Dipl. Kffr., Fachärztin für Chirurgie, Oberärztin Klinik für Orthopädie und Unfallchirurgie

\section{Frank J. P. Beeres}

Dr., MD. PhD, Facharzt für Chirurgie, Schwerpunkt Unfallchirurgie, ESBQ Trauma Surgery, Leitender Arzt Klinik Orthopädie und Unfallchirurgie

\section{Reto Babst}

Prof. Dr. med., Leiter Departement Chirurgie, Chefarzt Unfallchirurgie

\section{Björn-Christian Link}

Dr. med., Facharzt für Chirurgie, ESBQ Trauma Surgery, Facharzt für Orthopädie und Traumatologie des Bewegungsapparates, Leitender Arzt Klinik für Orthopädie und Unfallchirurgie
Korrespondenzadresse

Dr. med. Dipl. Kffr. Nadine Diwersi

Luzerner Kantonsspital Luzern

Spitalstrasse

CH 6000 Luzern 16

Tel.: + 41/412051111

nadine.diwersi@luks.ch

\section{Literatur}

[1] Hansmann C. Eine neue Methode der Fixierung der Fragmente bei komplizierten Frakturen. Verhandlungen der Deutschen Gesellschaft für Chirurgie 1886; 15: 134

[2] Texhammar R, Colton C. AO-Instrumente und -Implantate, Technisches Handbuch. Berlin, Heidelberg: Springer; 1994: 3

[3] Banks AS, Martin DE, McGlamry ED, Downey MS, Miller S]. Foot and Ankle Surgery. Philadelphia, PA: Lippincott Williams and Wilkins; 2001: 69

[4] de Marneffe R. Albin Lambotte, pioneer of modern osteosynthesis. Rev Med Brux 1982, 3: 493-495

[5] Synthes. LCP Proximale Femurplatte 4.5/5.5. Operationstechnik (11/2010), S. 14. Im Internet: http://synthes.vo.IInwd.net/ o16/LLNWMB8/INT\%20Mobile/Synthes\%20International/ Product\%20Support\%20Material/legacy_Synthes_PDF/ 016.000.403.pdf; Stand: 13.09.2018

[6] Müller ME, Allgöwer M, Schneider R, Willenegger H. Manual der Osteosynthese: AO-Technik. Berlin, Heidelberg: Springer; 1992: 54

[7] Jürgen Durst. Traumatologische Praxis: Standards für Diagnostik und Therapie für alle Fachgebiete. Stuttgart: Schattauer; 1997: 74

[8] Jürgen Durst. Traumatologische Praxis: Standards für Diagnostik und Therapie für alle Fachgebiete. Stuttgart: Schattauer; 1997: 72

[9] Hansis M, Weller S. Die operative Behandlung der Klavikulapseudarthrose. Operat Orthop Traumatol 1989; 1: 139-144

[10] Heim U. Die Pilon-tibial-Fraktur: Klassifikation, Operationstechnik, Ergebnisse. Berlin, Heidelberg: Springer; 1990: 152

Bibliografie

DOI https://doi.org/10.1055/a-0723-9470

Online-publiziert 14.12.2018 | OP-JOURNAL 2019; 35: 38-43

(C) Georg Thieme Verlag KG Stuttgart · New York

ISSN 0178-1715 